\title{
TEAM WORK FOR CONCURRENT ENGINEERING IN AGILENIRTUAL ENTERPRISE BY BM_VIRTUAL ENTERPRISE ARCHITECTURE REFERENCE MODEL
}

\author{
Antonio José Caulliraux Pithon ${ }^{\text {a) }}$, Goran D. Putnik ${ }^{\text {b) }}$ \\ a) Centro Federal de Educação Tecnológica - RJ, BRAZIL \\ pithon@cefet-ri.br,pithon@dps.uminho.pt \\ b) Universidade do Minho, Departamento de Produção e Sistemas, PORTUGAL \\ putnikgd@dps.uminho.pt
}

\begin{abstract}
It is presented an organizational structure for Concurrent Engineering $(C E)$ in Agile and/or Virtual Enterprise (AVE) based on the BM_Virtual Enterprise Architecture Reference Model (BM_VEARM). CE is a resume of a set of principles that includes teamwork, clients and supplier's involvement, parallel processes and continuous improvement of quality. On the other hand, an AVVE is seen as a species of short-term or long-term networked enterprise, with a dynamic structure, integrated over a universal set of resources, i.e. a global network of independent enterprises. The principal characteristic of the AVE organisational model presented is a broker as an agent of re-configurability of the CE teams. In accordance with the BM_VEARM, the broker is the principal agent of agility, and especially of virtuality.
\end{abstract}

Keywords. Concurrent Engineering, Virtual Enterprise, Teamwork, Broker, BM_Virtual Enterprise Architecture Reference Model

\section{INTRODUCTION}

At the present time, competitiveness can be interpreted as a principle requisite of an enterprise in which the satisfying of a market, demands adaptation to new organizational concepts and production systems, allied to an extremely efficient performance.

The model in development, proposes a new organizational architecture for EC based on BM_Virtual Enterprise Architecture Reference Model (BM_VEARM). This model must meet two basic principles. The first principle must satisfy the characteristics of virtual enterprises, i.e. the "open" architecture of the system, its capacity for "agility" (including re-configurability in real time) and its operation 
within an abstract environment, i.e. virtual. The second principle must meet the capacity to execute processes concurrently; this is the principal EC characteristic. In this new model, the broker is the principal agent of agility and virtuality, since he is the agent who provides greater flexibility to work groups. In other words, the model proposed should provide the highest flexibility of the CE teams in CE teams creation within the VE environment as well as in CE teams reconfiguration, in order to minimize eventual time losses and to maximize the response rapidness on client's demands.

This article will consist of three parts. The first will present the concept of a Virtual Enterprise according to the BM_VEARM model. In the second part, we approach the basic principles that guide the evolution of the work groups. In the last part, we present the EC model aimed at work groups within the Virtual Enterprise context, according to BM_VEARM.

\section{BM_VEARM VIRTUAL ENTERPRISE}

By BM_VEARM, The Virtual Enterprise model (Figure 1) is defined as a hierarchical model of multiple levels of the enterprise, with the broker inserted between two consecutive levels (principal/broker/agent) of the enterprise/manufacturing process control system, which ensures integrability, distributability, agility and virtuality.

Integrability is understood to be the capacity of an enterprise to access (interconnect) existing heterogeneous resources inside and outside the organization. The integration of heterogeneous resources should occur at low cost. This is a characteristic of open systems. Thus, the capacity to integrate is ensured by the architecture of open systems.

In the context of virtual enterprises, distributivity can be perceived as the capacity the enterprise has of integrating and operating needed resources at a distance. The concept of a competitive enterprise implies the ability to access the best resources: simply seeking the cooperation of other enterprises, purchasing components sub-contracting other companies or creating consortiums, as well as the capacity to manage all business and manufacturing functions, independent of distance, using Wide Area Network (WAN) technologies and corresponding protocols, e.g. Internet. Therefore, the distributed manufacture/enterprise system is defined as a system in which performance does not depend on the physical distance between the elements of the enterprise.

Speed is necessary for an enterprise to have rapid adaptability or rapid reconfigurability between two operations (off-line), in order to quickly respond to market changes. Virtuality is introduced with the objective of perfecting the performance of an agile enterprise, i.e. virtuality must furnish the system with the capacity of re-configurability during the intended operation (on-line) without the interruption of any other process. Virtuality, combined with agility, distributivity and integrability provides the enterprise with highest level of flexibility. The main agent of agility and virtuality is the broker and he can reconfigurate the organizational structure during an operation in real time or between two operations.

To synthesize what has been described above, we can now better define what to us is, a virtual enterprise (Putnik 2000): "The Virtual Enterprise (VE) is an optimized and synthesized enterprise on a universal set of resources with its physical 
structure substituted in real time. The project and control of the system is executed in an abstract or virtual environment".

"A set of universal resources" is understood to be any type of resource, primitive or complex, that can be distributed globally and can be located both inside and outside the frontiers of the enterprise. This implies a network, or consortium, of enterprises, i.e. networked enterprise over a globally distributed independent enterprises/partners in the VE.

The expression "its physical structure which can be substituted in real times" combined with "in a virtual environment" provides the high level of flexibility and agility that the enterprise requires. The specific architecture of the BM_VEARM, through the specific role of the broker, should provide the highest level of the VE reconfigurability dynamics (and, consequently, of the CE teams organization based on BM_VEARM). The broker acts as an interface between two hierarchical control layers in a way that one layer is hidden from the other and with the capability to reconfigure the VE architecture without interruption of the operation on the other control level, in order to avoid any loss of time due to the reconfiguration. The broker action between two operations, which obviously implies interruption of the process, is permitted by BM_VEARM but it is a relaxed case in terms of the VE, i.e. VE CE teams, reconfiguration dynamics requirements.

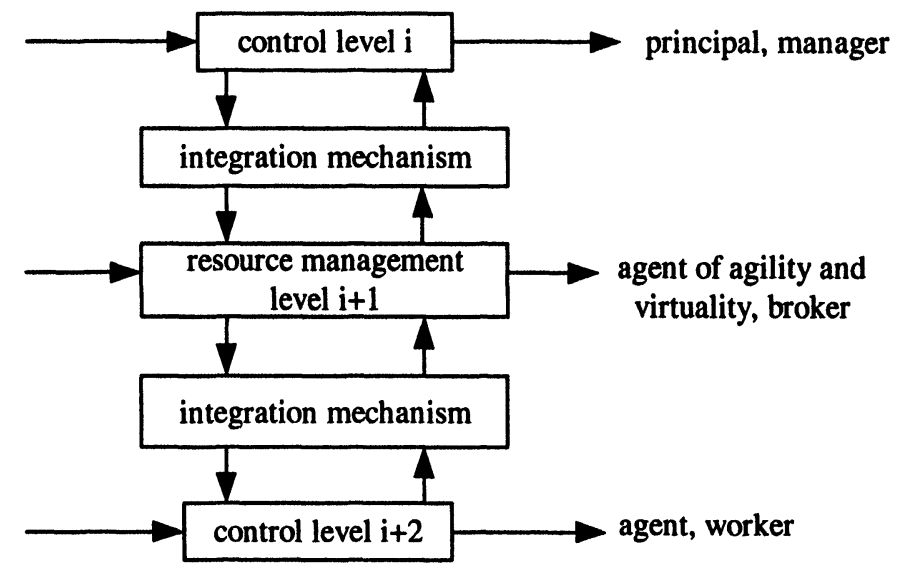

Figure 1 - Elementary hierarchical structure of Virtual Enterprise by BM_VEARM

\section{CONCURRENT ENGINEERING}

The concept of $\mathrm{CE}$ defines that various activities are developed in parallel, interactively, involving professionals from different specialties, covering the entire cycle of product development, in opposition to the traditional method of sequencing stages. Therefore, it becomes possible to re-feed an activity by others. This new form of working is very beneficial, since it avoids the possibility of a waste of time and resources, originating from a lack of complete involvement of the different sectors in all the stages contained in the project, besides improving the quality of development. On the contrary, the time and resources wasted in the execution of tasks that would later have to be redone will never be recovered (Pithon, Putnik; 2001). 


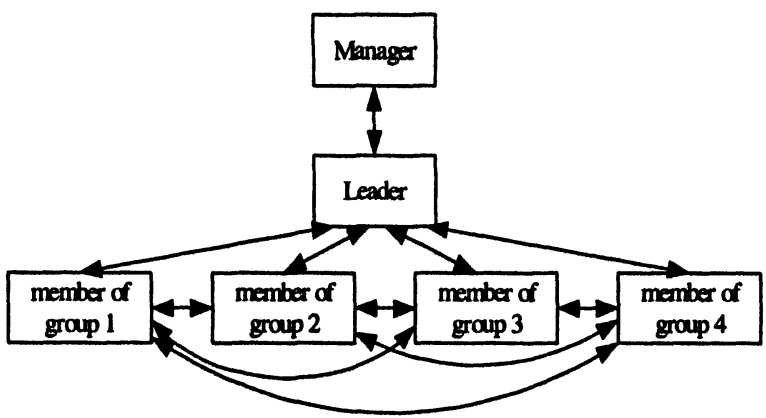

Figure 2 - Management Model of Concurrent Engineering Group management.

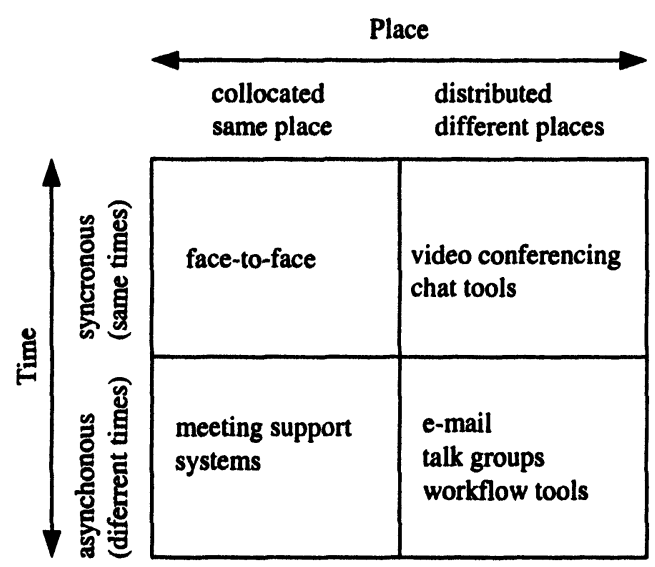

Figure 3 - Time/Place Matrix [Hawryszkiewycz, 1997]
The model that is being developed in this study, is guided by work groups known as the "task force" (Figure 2), which has as the leader as the linking element between the members of the group and the company board of directors, as well the group, i.e. the EC process

As support to activities, the group has a set of groupware tools available, which aid in communication, coordination and sharing of information between group members, so as to increase team productivity. These tools were grouped in Fig 3 in terms of time and place.

\subsection{Evolution of work Groups}

Over recent decades, the work group has suffered a number of transformations. They have evolved sequential work groups ("over-the-wall"), where activities are executed sequentially, passing through concurrent work groups, where activities are developed in parallel, moving on to virtual groups where group members are geographically dispersed and regroup through information technology (IT), including even virtual groups with the broker. This evolution can be followed in Figure 4.

(Yan 1999) cites agile CE groups as a manner of solving some existing CE traditional group problems. Still according to Yan, these groups have a better performance in relation to the traditional model because of resource sharing (the members of the groups are integrated by the same task), by the flexibility of organizing work groups (allowing them to be coordinated by themselves) and by the structural reorganization of the enterprise to permit small and medium enterprises to implement this model. The BM_VEARM model, described in Figure 5 and in Table 1 , considers all the proposals presented for this new agile EC work group and through the broker figure assumes the responsibility of re-configuring the group and guaranteeing the their agility. It is expected that our model will improve the 
flexibility and efficiency of the organization of the EC groups and as a consequence of their performance.

Before we present our model, we draw attention to the different approaches encountered in international literature in respect to virtual work groups. Various authors, Vários autores, (Pawar 2000), (Townsend 2000), (Ishaya 1999), (Cantu 1999), cite in their articles that virtual groups "are those in which their members appear geographically distributed....". Within the BM_VEARM concept, we understand that these groups are considered as distributed groups and cannot be considered virtual groups only because they are geographically distributed, since in terms of our concept, virtuality is not synonymous with distributability or the use of electronic means.

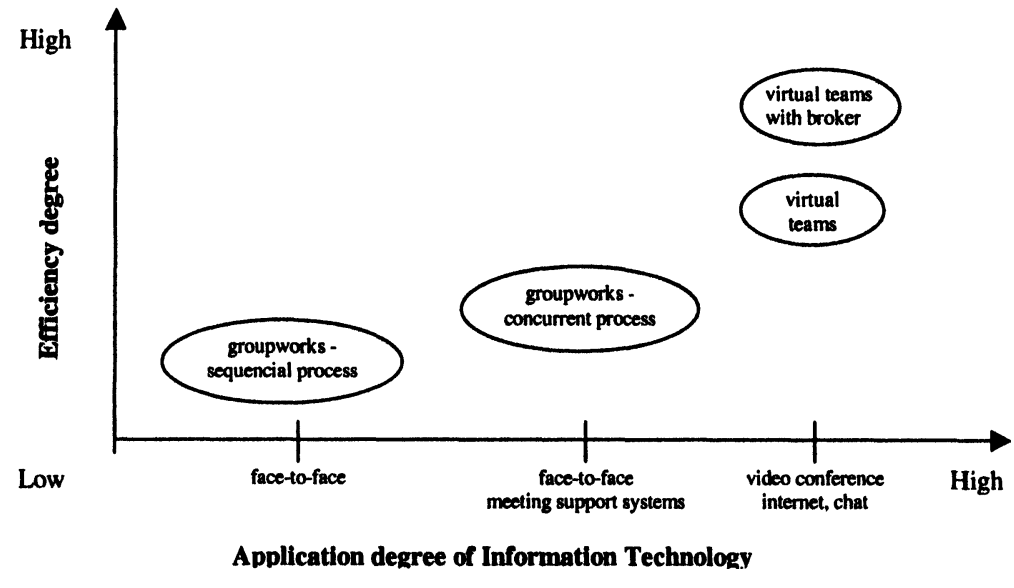

Figure 4 Evolution of Work groups

\section{EC WORK TEAMS FOR BM-VEARM}

As was described in item 2, in BM_VEARM Virtual Enterprise the broker figures as the linking agent between two consecutive hierarchical levels.

In Item 3, it was stated that the CE acts with work groups interacting mutually so as to reduce the new product development time.

Aiming at the interaction of the CE with the VE by BM-VEARM, we are presenting the model of the CE groups by BM_VEARM.

These groups consist of the Manager, Leader, Broker and Group members, having the following functions:

- Manager: in charge of all operations at the macro level and for action strategy of the enterprise. The manager has global supervision, delegating lesser and detailed responsibilities to the leaders;

- Leader: in charge of the execution of tasks in details and coordination of work groups;

- Broker: the element selected by management/leader to obtain necessary resources for the execution of tasks, integrating selected resources, dynamically reconfigurating $\mathrm{CE}$ resources.

- Group members: components that form work groups. They execute the tasks.

This model considers two distinct variants: 
a) CE Agile groups - In this situation the broker reconfigures the member of the work group in off-line. i.e., interrupting a given operation, in accordance with the leader, or operating between 2 distinct operations. In the agile work groups, a member of the group can interact with a colleague using the communication tools that are at his disposal (Figure 3), i.e. the member of the group maintain direct among themselves when they are interacting (see Figure 5); this relationship between a member of the group and other group members is 1:n, i.e. they all communicate with each other (Figure 5 presents the other relationships between the members of the agile groups).

b) CE Virtual groups - In this situation the broker reconfigures the on-line group members, i.e. without interrupting the activities of a given operation. The virtuality of this model consists of as substitution of one or more group members without the substitution being perceived by the other group members or by another hierarchically higher level (Figure 6). In the virtual work groups the group members do not integact directly with each other, these communicate among themselves indirectly through the broker who provides the interface (among other brokerage functions) by means of a communication software e.g. chat with an animated screen where the animation represents any member (virtual) of the group. The broker and group members do not know each other. The relationship of each group member with the broker is 1:1, as is the case of the broker's relationship with the leader of the group and the manager.

BM_VEARM work groups present some problems. The performance of the group is directly related to the social activities carried out inside and outside the work and how a member of the group does not "see" and has no direct contact with a colleague, makes it difficult to maintain a social relationship (e.g. lack of dialogue, little or almost no exchange of information between them), resulting in a low performance level of the group. Another problem that affects the group is the use by part of group members of different work platforms/systems (e.g. different versions of communication systems) thereby making them incompatible or costly.

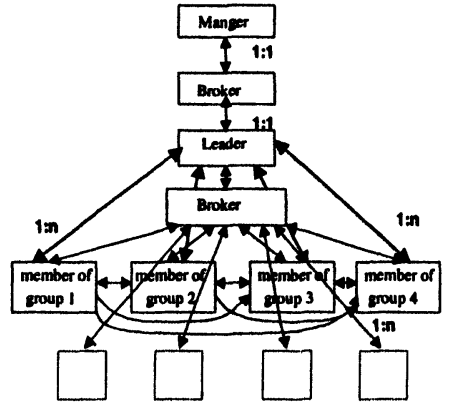

Figure 5 Management Model of Agile Groups

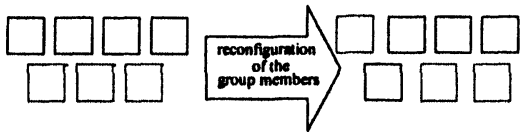

Figure 5a Reconfiguration of the Group Members

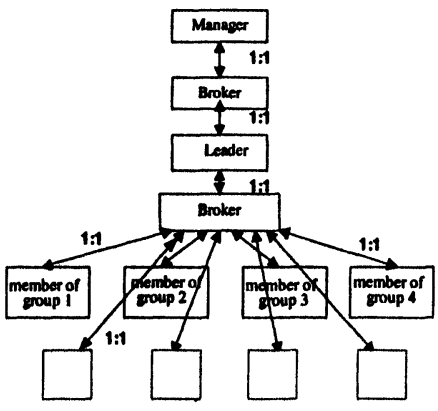

Figure 6 Management Model of Virtual Groups

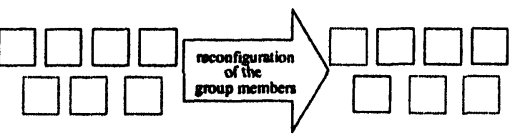

Figure 6a Reconfiguration of the Group Members 
Table 1 - Comparison between work groups

\begin{tabular}{|l|l|l|l|}
\cline { 2 - 4 } \multicolumn{1}{c|}{} & $\begin{array}{l}\text { Distributed Virtual } \\
\text { Groups described in } \\
\text { literature }\end{array}$ & $\begin{array}{l}\text { CE Agile Groups } \\
\text { according to } \\
\text { BM-VEARM }\end{array}$ & $\begin{array}{l}\text { CE Virtual Groups } \\
\text { according to } \\
\text { BM-VEARM }\end{array}$ \\
\hline Virtuality & not present & not present & is present \\
\hline Flexibility & low flexibility & Average/high flexibility & highest flexibility \\
\hline Reconfigurability & $\begin{array}{l}\text { does not permit } \\
\text { reconfigurability during } \\
\text { project }\end{array}$ & $\begin{array}{l}\text { permits } \\
\text { reconfigurability during } \\
\text { project with interruption } \\
\text { of EC process }\end{array}$ & $\begin{array}{l}\text { permits reconfigurability } \\
\text { during project without } \\
\text { interruption of the EC } \\
\text { process }\end{array}$ \\
\hline tools & $\begin{array}{l}\text { face-to-face through } \\
\text { video conference }\end{array}$ & $\begin{array}{l}\text { face-to-face through } \\
\text { video conference }\end{array}$ & $\begin{array}{l}\text { uses VR conference once a } \\
\text { member does not know his } \\
\text { colleague nor notices his } \\
\text { substitution }\end{array}$ \\
\hline Applicability & present & present/future & future \\
\hline
\end{tabular}

The implementation of these groups requires a profound reformation in the organizational structure/culture of the enterprise, beginning with communication structures (computers, networks, communication systems), which must be prepared for high-speed data and video work.

Below is a summary of the principal advantages and disadvantages found in virtual groups in relation to other groups.

\section{Table 2 - BM_VEARM vs. Collocated Teams}

\begin{tabular}{|c|c|}
\hline Advantages/Benefits & Disadvantage/Problems \\
\hline $\begin{array}{l}\text { - the broker does not need to interrupt an activity } \\
\text { to substitute a member of the group by } \\
\text { another. } \\
\text { - a systematic search for reduction of EC } \\
\text { process costs by the broker, always aiming at } \\
\text { an increase of efficiency in these processes. } \\
\text { - the productivity of the group will always be } \\
\text { maintained or improved, as the broker } \\
\text { substitutes a member of the team by another } \\
\text { having the same condition, without the final } \\
\text { task being affected } \\
\text { - robustness, quality }\end{array}$ & $\begin{array}{l}\text { - accentuated incompatibility between product } \\
\text { development platforms (e.g. CAD systems, } \\
\text { video-conference systems) } \\
\text { - low group performance due to the members } \\
\text { being dispersed and having little contact } \\
\text { among themselves } \\
\text { - reconfiguration costs and broker costs } \\
\text { - management complexity } \\
\text { - lack of knowledge } \\
\text { - inadequate enterprise organization } \\
\text { - management complexity }\end{array}$ \\
\hline
\end{tabular}

\section{CONCLUSION}

It is expected that work groups with a broker inserted at principal levels and agent present a better performance in relation to other models and that the problems described above related to virtual groups are solved.

With this new proposal we intend to:

- increase the efficacy of the group in the development of new tasks arising from the increased competence of the individuals;

- maintain productivity, quality, agility of the group by means of broker action. 
The next stage is the construction of a prototype that will serve as a demonstrator of the implementation of this model and for the model validation.

\section{Footnotes}

1) A resource is (from an enterprise point of view) an object that it uses to conduct or support the execution of one or more processes (e.g. materials, machines, tools, computers, human operators, time, money, software, etc.). An enterprise is also a resource if it is contracted by another enterprise to render a service. In this work, a member of the group is a resource.

\section{Acknowledgements}

We would like to thank FCT (Fundação para a Ciência e a Tecnologia - The Foundation for Science and Technology) of Portugal, for the financial support provided for this research.

\section{REFERENCES}

Cantu, Cyntia (1999) Virtual Teams, Center for the Study of Work Teams (CSWT), University of North Texas

Hawryszkiewycz, Igor (1997) Designing the Networked Enterprise, Artech House

Ishaya, Tanko, Macaulay, Linda (1999) The Role of Trust in Virtual Teams, Proceedings of the $2^{\text {nd }}$ International VoNet - Workshop, Zurich, September 23-24

Pawar, K. S., Sudi (2000) Virtual collocation of design teams: coordination for speed, International Journal of Agile Management Systems, Vol. 2, n²: 104-113

Pithon A., Putnik G. (2001) Concurrent Engineering and Groupwork - Introduction, Technical Report, CESP-GIS-01-01, University of Minho

Putnik G. D. (2000) BM_Virtual Enterprise Architecture Reference Model, In A. Gunasekaran (Ed.) Agile Maniufacturing: 21st Century Manufacturing Strategy, Elsevier Science Publ., pp. 73-93.

Townsend, A.M. D., Samuel, Hendricson, Anthony (2000) Virtual Teams: Technology and the Workplace of the Future, IEEE Engineering Management Review, MCB University Press, Vol. 28, $n^{\circ} 10$ : 69-80

Yan, H. S. (1999) Agile Concurrent Engineering, Integrated Manufacturing Systems, MCB University Press, Vol. 10, n $^{\circ}$ 2: 103-1 